\title{
Traumatismo torácico contuso
}

\author{
ROBERTO GONZÁLEZ L.***, ALEJANDRA RIQUELME U.***, CLAUDIO TOLOZA A.****, \\ RODRIGO REYES M.**,*****, ENRIQUE SEGUEL S.*******, ALECK STOCKINS L.**,*****, \\ ANDRÉS JADUE T.*****, MATÍAS ÁVALOS T.****, SEBASTIÁN BARRA M.****, FELIPE ALARCÓN \\ O.**** y EMILIO ALARCÓN C.***
}

\section{Blunt Thoracic Trauma}

Background: Thoracic trauma (TT) is a major cause of morbimortality, involved in $25-50 \%$ of trauma deaths. Internationally, blunt thoracic trauma (BTT) is the most frequent type of TT. Objective: Our objectives are to describe the clinical characteristics, treatments, morbidity and mortality in patients hospitalized by blunt thoracic trauma (BTT) in our institution. Material and Methods: Crosssectional descriptive study from january-1981 to december-2017. Prospective database review, surgical protocols and clinical files. The characteristics of the BTT are described and compared. The following trauma severity indices (TSI) were calculated: Injury Severity Score (ISS), Revised Trauma Score (RTST) and Trauma Injury Severity Score (TRISS). Results: 4,163 patients were hospitalized because of TT, $1.719(41.3 \%)$ of them with BTT. 1,327 (77.2\%) patients were men, average age $46.7 \pm 18.8$ years-old. We considered isolated TT 966 (56.2\%), associated with extrathoracic lesions $753(43,8 \%)$ and 508 (29.6\%)with polytraumatism. Mechanism: Traffic accident 838 (48.7\%), fall down from a height 279 (16.2\%). Lesions and intrathoracic findings: rib fracture 1.294 (75.3\%), pneumothorax 752 (43.7\%). Treatment: Medical 876 (50.8\%), pleurotomy 704 (41\%) and thoracic surgery 141 (8.2\%). Average hospitalized period $9.2 \pm 9.5$ days. According to TSI: ISS average $14.1 \pm 11.1$, RTS-T average $11.5 \pm$ 1.5, TRISS average 6.6. Morbidity in 297 (17.3\%), mortality in 68 (4\%). Discussion: The TTC was mainly attributed to the traffic accident. Rib fracture was the most common chest injury. The majority of patients required only medical treatment. Mortality was lower than expected according to TSI.

Key words: Thoracic injury; rib fractures; pneumothorax; thoracic surgery; injury severity score; accidents, traffic.

\section{Resumen}

Introducción: El traumatismo torácico (TT) es una causa importante de morbilidad y mortalidad, presente en el $25-50 \%$ de la mortalidad por traumatismo. El TT contuso (TTC) es el tipo más frecuente de TT según las diferentes publicaciones internacionales. Objetivo: Nuestros objetivos son describir las características, tratamientos, morbilidad y mortalidad en pacientes hospitalizados por TTC en nuestra institución. Material y Métodos: Estudio descriptivo transversal desde enero-1981 a diciembre-2017. Revisión de una base de datos prospectiva, protocolos quirúrgicos y fichas clínicas. Se describen y comparan las características de los TTC. Se calcularon índices de gravedad de traumatismo (IGT): Injury Severity Score (ISS), Revised Trauma Score (RTS-T), Trauma Injury Severity Score (TRISS). Resultados: Total 4.163 pacientes hospitalizados por TT, 1.719 (41,3\%) TTC. Hombres 1.327 (77,2\%), edad promedio 46,7 $\pm 18,8$ años. Se consideró TT aislado $966(56,2 \%)$, asociado a lesiones extratorácicas $753(43,8 \%)$ y de estos 508 (29,6\%) eran politraumatizados. Mecanismo: Accidente de tránsito 838 (48,7\%), caída de altura 279 (16,2\%). Lesiones y hallazgos torácicos: fractura costal

\footnotetext{
* Cirujano Cardiotorácico, Centro Cardiovascular, Hospital Clínico Regional de Concepción "Dr. Guillermo Grant Benavente". Concepción, Chile.

** Departamento de Cirugía, Facultad de Medicina, Universidad de Concepción. Concepción, Chile.

*** Posbecada Cirugía General, Facultad de Medicina, Departamento de Cirugía, Universidad de Concepción. Concepción, Chile.

**** Alumno de la Carrera de Medicina, Facultad de Medicina, Universidad de Concepción. Concepción, Chile.

***** Cirujano Cardiovascular, Centro Cardiovascular, Hospital Clínico Regional de Concepción "Dr. Guillermo Grant Benavente". Concepción, Chile.
} 
1.294 (75,3\%), neumotórax 752 (43,1\%). Tratamiento: médico 874 (50,8\%), pleurotomía 704 (41\%) y cirugía torácica 141 (8,2\%). Periodo de hospitalización 9,2 \pm 9,5 días. Según IGT: ISS promedio 14,1 $\pm 11,1$, RTS-T promedio 11,5 \pm 1,5, TRISS promedio 6,6. Morbilidad en 297 (17,3\%), mortalidad en 68 (4\%). Discusión: La causa principal de los TTC fue el accidente de tránsito. La fractura costal correspondió a la lesión torácica más frecuente. La mayoría requirió solo tratamiento médico. La mortalidad fue menor a la esperada según IGT.

Palabras clave: Traumatismo torácico; fractura costal; neumotórax; cirugía torácica; puntaje de gravedad del daño; accidentes del tránsito.

\section{Introducción}

El traumatismo es motivo frecuente de consulta en los servicios de urgencias en todo el mundo. Su impacto es tal, que se constituye una de las principales causas de mortalidad global. Además, si nos remitiéramos solo a la población menor de 40 años, los traumatismos lideran las causas de fallecimiento en este grupo ${ }^{1}$. El traumatismo torácico (TT) es responsable de más del 25\% de muertes traumáticas en Estados Unidos, contribuyendo en otro $50 \%$ de forma importante ${ }^{2,3}$. El TT se puede clasificar según su mecanismo en contuso o penetrante, variando sus respectivas frecuencias según el lugar geográfico. El traumatismo torácico contuso (TTC) se puede definir como aquel que provoca lesiones torácicas producto del contacto directo con un objeto contundente $^{4}$. Por su parte, el TTC, al igual que el TT en general, usualmente no requiere alguna intervención quirúrgica mayor, evolucionando favorablemente hasta en un $85 \%$ al ser tratado con pleurotomía y/o médicamente ${ }^{5}$. Sin embargo, en los politraumatizados, dada la presencia de lesiones graves que elevan considerablemente el riesgo de mortalidad, es más frecuente la necesidad de tratamientos invasivos ${ }^{1,3,6}$. Series internacionales revelan una mortalidad global por TTC cercana al $10 \%{ }^{3}$. Estudios aislados describen distintas características clínicas y epidemiológicas del TTC según el sitio geográfico, por lo que es fundamental aportar con información sobre los TTC en nuestro medio ${ }^{5-7}$.

Nuestros objetivos son describir las características, tratamientos, morbilidad y mortalidad en pacientes hospitalizados por TTC.

\section{Material y Métodos}

Se realizó estudio descriptivo analítico con los pacientes hospitalizados por TTC en el Hospital Clínico Regional "Dr. Guillermo Grant Benavente" de Concepción-Chile, tratados por el Equipo de Cirugía Cardiotorácica, en el período comprendido entre enero de 1981 y diciembre de 2017. La información se obtuvo a partir protocolos prospectivos de TT realizados al momento de ingreso al hospital, revisión de base de datos, protocolos quirúrgicos y fichas clínicas. Se ordenaron los datos mediante planilla Microsoft Excel ${ }^{\circledR}$ y se utilizó el programa SSPS $24^{\circledR}$ con función ANOVA de un factor para análisis estadístico con 3 variables cuantitativas y prueba chi cuadrado $\left(\mathrm{X}^{2}\right)$ para variables cualitativas, considerándose significativo $\mathrm{p}<0,05$. Se describen edad, sexo, características clínicas, mecanismos, tratamiento, morbilidad, mortalidad e índices de gravedad de traumatismo (IGT) como: ISS $^{8}$ (Injury Severity Score), RTS-T ${ }^{9}$ (Revised Trauma Score Triage) y TRISS ${ }^{10}$ (Trauma Injurity Severity Score). El ISS determina gravedad de acuerdo a lesión anatómica, el RTS-T de acuerdo a lesión fisiológica (presión arterial sistólica, frecuencia respiratoria y escala de Glasgow) y el TRISS engloba los dos anteriores y los relaciona con la edad del paciente y el mecanismo del traumatismo, determinando el riesgo de mortalidad. Se consideró politraumatizado un ISS $>16$ puntos $^{11}$. Se compararon las características de los grupos de TT aislado con el TT con lesiones asociadas y dentro de éste, el subgrupo de los politraumatizados.

El estudio y análisis de las hospitalizaciones por traumatismo torácico fue autorizado por el Comité de Ética de nuestra institución.

\section{Resultados}

Durante el período se hospitalizaron 4.163 TT, de los cuales 1.735 fueron TTC. Entre estos se excluyeron $16(0,9 \%)$ por ser menores de 15 años, quedando un total de 1.719 pacientes.

Fueron hombres 1.327 (77,2\%) y mujeres 392 $(22,8 \%)$, con una relación hombre/mujer de 3,4/1. La edad promedio, en años, fue de 46,7 $\pm 18,8$, mediana 45 (rango 15-103).

El mecanismo más frecuente fue el accidente de tránsito en $838(48,7 \%)$ casos, seguido de accidente doméstico en 291 (16,9\%) pacientes, lo 
que se observa en la Tabla 1.

El principal lugar del traumatismo fue la vía pública en $1.185(68,9 \%)$ casos. Los agentes principales del TTC fueron los vehículos motorizados en $836(48,6 \%)$ casos y objetos contundentes en 309 (18\%). Según el tipo de traumatismo, se consideró traumatismo torácico aislado en 966 $(56,2 \%)$ y con lesiones asociadas $753(43,8 \%)$, siendo politraumatizados $508(29,6 \%)$ casos (Tabla 2).

Entre las lesiones y hallazgos torácicos más frecuentes se encuentran: fracturas costales en $1.294(75,3 \%)$ y neumotórax en $752(43,7 \%)$ (Tabla 3). De los pacientes con fracturas costales, más de la mitad presentó alguna complicación torácica. En relación a las lesiones asociadas extratorácicas, las que predominaron fueron las craneoencefálicas en $401(23,3 \%)$ pacientes (Tabla 4).

El tratamiento definitivo fue médico exclusivo en $874(50,8 \%)$ casos, requiriendo un tratamiento invasivo torácico en $845(49,2 \%)$ pacientes, de los cuales se realizaron $141(8,1 \%)$ cirugías torácicas. En relación a la temporalidad de las cirugías torácicas, se realizaron en su mayoría de manera diferida ( $>24 \mathrm{~h}$ ) en el 53,2\% (75 casos), seguidas por las cirugías urgentes $(\leq 4 \mathrm{~h})$ en el 45,4\% (64 casos) (Tabla 5).

Del total de los pacientes con TTC, 297 $(17,3 \%)$ presentaron morbilidad, requirieron reoperación torácica $5(0,3 \%)$ pacientes y la mortalidad fue de 68 (4\%) pacientes. El tiempo promedio de estadía hospitalaria fue de 9,2 \pm 9,5 días (Tabla 6).

Dentro de los pacientes fallecidos, la principal causa fue el Síndrome de Respuesta Inflamatoria Sistémica en $36(2,1 \%)$ casos, lo que equivale al $52,9 \%$ de las causas de mortalidad. Dentro de la primera semana post traumatismo, fallecieron 38 pacientes, lo que equivale al $55,9 \%$ de los fallecidos por TTC. En cuanto a los índices de gravedad del traumatismo, en nuestra serie de pacientes con TTC hubo un promedio de ISS de $14,1 \pm 11,1$, RTS-T de $11,5 \pm 1,5$ y TRISS 6,6 ; siendo la mortalidad observada menor que la esperada (Tabla 7).

Las características principales al dividir los TTC según tipo de traumatismo en aislados y con lesiones asociadas y dentro de estos, el subgrupo de politraumatizados, se presentan en la Tabla 8 , observándose diferencias estadísticamente significativas entre estos tres grupos de pacientes.
Tabla 1. Edad, sexo y mecanismo del traumatismo en pacientes hospitalizados por traumatismo torácico contuso

Total TTC (1.719)

\begin{tabular}{|lc|}
\hline & Total TTC (1.719) \\
Edad años (promedio) & $46,7 \pm 18,8$ \\
Mediana & 45 \\
Rango & $15-103$ \\
$\geq 60$ años & $456(26,5 \%)$ \\
Sexo & \\
Hombre & $1.327(77,2 \%)$ \\
Mujer & $392(22,8 \%)$ \\
Mecanismo & \\
Accidente de tránsito & $838(48,7 \%)$ \\
Accidente doméstico & $291(16,9 \%)$ \\
Agresión & $208(12,1 \%)$ \\
Accidente laboral & $114(6,6 \%)$ \\
Otro mecanismo & $268(15,6 \%)$ \\
\hline
\end{tabular}

TTC: Traumatismo torácico contuso.

Tabla 2. Lugar, agente y tipo de traumatismo en pacientes hospitalizados por traumatismo torácico contuso

Total TTC (1.719)

\section{Lugar del traumatismo}

$\begin{array}{lc}\text { Vía pública } & 1.185(68,9 \%) \\ \text { Domicilio } & 316(18,4 \%) \\ \text { Otros } & 218(12,7 \%) \\ \text { Agente del traumatismo } & \\ \text { Vehículo motorizado } & 836(48,6 \%) \\ \text { Objeto contundente } & 309(18 \%) \\ \text { Caída de altura } & 279(16,2 \%) \\ \text { Caída a nivel } & 241(14 \%) \\ \text { Vehículo no motorizado } & 26(1,5 \%) \\ \text { Animal } & 13(0,8 \%) \\ \text { Otro agente } & 15(0,9 \%)\end{array}$

Tipo de TT

TT asilado

$966(56,2 \%)$

TT con lesiones asociadas

$753(43,8 \%)$

Politraumatismo

$508(29,6 \%)$

TTC: Traumatismo torácico contuso. TT: Traumatismo torácico. 
Tabla 3. Hallazgos y lesiones torácicas en pacientes hospitalizados por traumatismo torácico contuso

Total TTC (1.719)

\section{Hallazgos y lesiones}

Fracturas costales

$1.294(75,3 \%)$

Neumotórax

$752(43,7 \%)$

Hemotórax no masivo

$666(38,7 \%)$

Contusión pulmonar

$503(29,3 \%)$

Enfisema subcutáneo

$439(25,5 \%)$

Fractura y/o luxación esternal

$131(7,6 \%)$

Lesión diafragmática

$25(1,5 \%)$

$21(1,2 \%)$

$160,9 \%)$

$12(0,7 \%)$

$8(0,5 \%)$

$5(0,3 \%)$

$3(0,2 \%)$

TTC: Traumatismo torácico contuso.

Tabla 4. Fracturas costales y lesiones extratorácicas asociadas en relación al total de pacientes hospitalizados por traumatismo torácico contuso

Total TTC (1.719)

$\begin{array}{lc}\text { Fracturas costales } & 1.294(75,3 \%) \\ 1 \text { a } 3 \text { costillas } & 626(36,4 \%) \\ 4 \text { a } 6 \text { costilas } & 460(26,8 \%) \\ >6 \text { costillas } & 207(12,0 \%) \\ \text { Tórax volante } & 194(11,3 \%) \\ \text { Fracturas complicadas } & 974(56,7 \%) \\ \text { FC con complicaciones torácicas } & 949(55,2 \%) \\ \text { FC con complicaciones } & \\ \text { extratorácicas } & 152(8,8 \%) \\ \text { Lesiones asociadas } & \\ \text { Craneoencefálicas } & 401(23,3 \%) \\ \text { Extremidades } & 284(16,5 \%) \\ \text { Abdominales } & 225(13,1 \%) \\ \text { Fractura de pelvis } & 128(7,4 \%) \\ \text { Faciales } & 88(5,1 \%) \\ \text { Partes blandas } & 57(3,3 \%)\end{array}$

FC: Fracturas costales. TTC: Traumatismo torácico contuso.
Tabla 5. Tratamiento torácico definitivo realizado y temporalidad de las cirugías torácicas en pacientes hospitalizados por traumatismo torácico contuso

$\begin{array}{lc}\text { Tratamiento médico exclusivo } & \mathbf{8 7 4}(\mathbf{5 0 , 8} \%) \\ \text { Tratamiento invasivo torácico } & 845(49,2 \%) \\ \text { Pleurotomía exclusiva } & 704(41 \%) \\ \text { Cirugía torácica } & 141(8,2 \%)\end{array}$

Temporalidad de las cirugías torácicas
Urgentes $(\leq 4 \mathrm{~h})$
$64(3,7 \%)$
Precoces $(>4-24 \mathrm{~h})$
$2(0,1 \%)$
Diferidas $(>24 \mathrm{~h})$
$75(4,4 \%)$

Tabla 6. Morbilidad, mortalidad y estadía hospitalaria en pacientes hospitalizados por traumatismo torácico contuso

\begin{tabular}{lc|}
\hline Morbilidad & Total TTC (1.719) \\
Reintervenciones & $\mathbf{2 9 7}(\mathbf{1 7 , 3 \% )}$ \\
Extratorácicas & $20(1,2 \%)$ \\
Torácicas & $15(0,8 \%)$ \\
Mortalidad & $5(0,4 \%)$ \\
TT aislado & $\mathbf{6 8}(\mathbf{4 , 0 \% )}$ \\
TT con lesiones asociadas & $8(0,8 \%)$ \\
Politraumatismo & $60(8,0 \%)$ \\
Estadía hospitalaria en días & $59(11,4 \%)$ \\
Promedio & $9,2 \pm 9,5$ \\
Mediana & 6 \\
Rango & $1-91$ \\
\hline
\end{tabular}

TT: Traumatismo torácico. TTC: Traumatismo torácico contuso.

Tabla 7. Causas y temporalidad de la mortalidad e índices de gravedad del traumatismo en pacientes hospitalizados por traumatismo torácico contuso

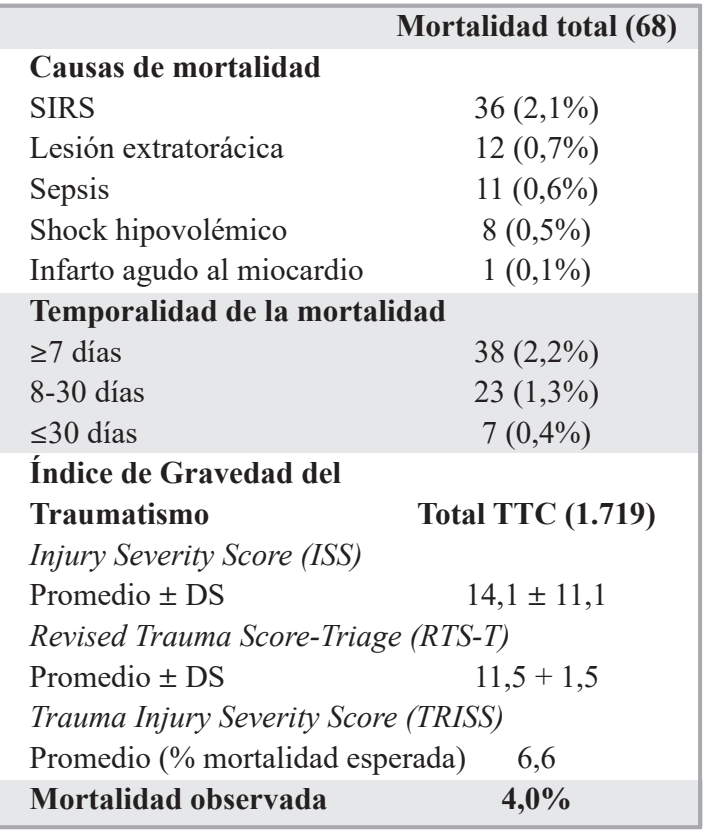

SIRS: Síndrome de respuesta inflamatoria sistémica. 
Tabla 8. Caracterización según tipo de traumatismo en pacientes hospitalizados por traumatismo torácico contuso (TTC)

\begin{tabular}{|c|c|c|c|c|}
\hline & $\begin{array}{c}\text { TTC aislado } \\
\text { (966) }\end{array}$ & $\begin{array}{l}\text { TTC con lesiones } \\
\text { asociadas } \\
(753)\end{array}$ & $\begin{array}{c}\text { TTC con lesiones } \\
\text { asociadas } \\
\text { subgrupo PT } \\
(\mathbf{7 5 3} / \mathbf{5 0 8 )}\end{array}$ & $\begin{array}{l}\text { Valor de } \\
\quad \text { p }\end{array}$ \\
\hline $\begin{array}{l}\text { Características } \\
\text { Edad años (promedio) } \\
\text { Mediana } \\
\text { Rango } \\
\geq 60 \text { años }\end{array}$ & $\begin{array}{c}49,2 \pm 19,5 \\
49 \\
15-103 \\
302(31,2 \%)\end{array}$ & $\begin{array}{c}43,5 \pm 17,3 \\
42 \\
15-95 \\
153(20,1 \%)\end{array}$ & $\begin{array}{c}42,4 \pm 17 \\
41 \\
15-68 \\
91(17,9 \%)\end{array}$ & $\begin{array}{l}<0,001 \\
<0,001\end{array}$ \\
\hline $\begin{array}{l}\text { Principales mecanismos } \\
\text { Accidente de tránsito } \\
\text { Accidente doméstico } \\
\text { Agresión }\end{array}$ & $\begin{array}{l}274(28,3 \%) \\
253(26,2 \%) \\
170(17,6 \%)\end{array}$ & $\begin{array}{l}565(74 \%) \\
38(5 \%) \\
41(5,4 \%)\end{array}$ & $\begin{array}{c}406(79,9 \%) \\
15(3 \%) \\
16(3,1 \%)\end{array}$ & $\begin{array}{l}<0,001 \\
<0,001 \\
<0,001\end{array}$ \\
\hline $\begin{array}{l}\text { Tratamiento invasivo } \\
\text { Pleurotomía exclusiva } \\
\text { Cirugía torácica }\end{array}$ & $\begin{array}{c}327(33,9 \%) \\
65(6,7 \%)\end{array}$ & $\begin{array}{c}368(48,9 \%) \\
76(10,1 \%)\end{array}$ & $\begin{array}{c}282(55,5 \%) \\
58(11,4 \%)\end{array}$ & $\begin{array}{c}<0,001 \\
0,004\end{array}$ \\
\hline Médico exclusivo & $574(59,4 \%)$ & $309(41 \%)$ & $168(33,1 \%)$ & $<0,001$ \\
\hline $\begin{array}{l}\text { Estadía hospitalaria } \\
\text { Promedio (días) }\end{array}$ & $6,8 \pm 6,3$ & $12,2 \pm 11,7$ & $14,1 \pm 12,8$ & $<0,001$ \\
\hline Morbilidad & $102(10,6 \%)$ & $195(25,9 \%)$ & $164(32,3 \%)$ & $<0,001$ \\
\hline $\begin{array}{l}\text { IGT } \\
\text { Injury severity score (ISS) } \\
\text { Promedio }\end{array}$ & $7,8 \pm 4,8$ & $22,1 \pm 11,5$ & $27,6 \pm 10,1$ & $<0,001$ \\
\hline $\begin{array}{l}\text { Revised trauma score } T \text { (RTS-T) } \\
\text { Promedio }\end{array}$ & $11,9 \pm 0,6$ & $10,9 \pm 2,1$ & $10,5 \pm 2,4$ & $<0,001$ \\
\hline $\begin{array}{l}\text { Trauma injury severity score (TRIS } \\
\text { Promedio (\% mortalidad esperada) }\end{array}$ & SS) & 11,9 & 16,9 & 0,013 \\
\hline Mortalidad observada & $0,8 \%$ & $7,7 \%$ & $11,4 \%$ & $<0,001$ \\
\hline
\end{tabular}

PT: politraumatismo. IGT: Índices de gravedad del traumatismo.

\section{Discusión}

El TT es un problema siempre vigente por su complejidad fisiopatológica, independiente del mecanismo y agente causal ${ }^{12}$. Existe un número importante de publicaciones a nivel mundial sobre traumatismo y TT, principalmente en Norteamérica y Europa. Lamentablemente, en Chile, es escasa la información objetiva que se tiene acerca de TT y, en particular, del grupo de los TTC. Por lo tanto, el presente estudio busca revelar y describir más profundamente las características de este grupo de pacientes.

Según la ley de Newton "un cuerpo en reposo permanecerá en reposo y un cuerpo en movimiento permanecerá en movimiento a menos que una fuerza externa actúe sobre él". El comienzo o interrupción brusca del movimiento, causa daño ya que "la energía no puede ser creada ni destruida, sino que únicamente se transforma". La energía cinética (EC), por su parte, depende de la masa (M) y la velocidad (V) según la ecuación:

$$
\mathrm{EC}=1 / 2\left(\mathrm{M} \cdot \mathrm{V}^{2}\right)
$$

De esta ecuación se concluye que el principal elemento que actuará e influirá en el traumatismo, y en particular, en el contuso, es la velocidad a la que ocurre. Esto adquiere más importancia aún en nuestra serie, ya que el mecanismo predominante fue el accidente de tránsito por vehículos motorizados que circulan generalmente a alta velocidad, transmitiendo grandes fuerzas de desaceleración. Es así como las estructuras torácicas, son expuestas a compresión generando principalmente fracturas costales, tórax volante, contusiones 
cardíacas y pulmonares y a desaceleración causando lesiones por cizallamiento, principalmente a nivel de la aorta. También se puede generar el fenómeno denominado cavitación, forzando a los tejidos a localizarse fuera de su lugar habitual, siendo mayor en aquellos tejidos más densos ${ }^{13}$. Se desprende de lo anterior, que la gravedad del traumatismo va a depender en forma importante del mecanismo (por la energía involucrada), y de otros factores como la edad y las comorbilidades asociadas ${ }^{6}$. peos y estadounidenses de TT, muestran que los TTC son más frecuentes que los penetrantes ${ }^{14,15}$, incluso en un estudio canadiense se llegó a un $90 \%$ de frecuencia de los $\mathrm{TTC}^{16}$. Sin embargo, en nuestra serie, los TTC ocupan el $41,7 \%$ de los casos, lo que concuerda con distintas series latinoamericanas en las que predomina el TT penetrante versus el contuso ${ }^{5}$. Está documentado que los TT ocurren principalmente en hombres jóvenes alcanzando frecuencias de hasta $94 \%$ en algunas series, con edades promedio cercanas a los 30 años $^{5}$. Sin embargo, en nuestra revisión, se puede apreciar un promedio de edad que supera los 46 años, dado principalmente por un número importante de adultos mayores $(26,5 \%)$, grupo en el cual predominan los TTC.

Casi la mitad de los TTC, ocurrieron producto de un accidente de tránsito por vehículos motorizados. Está descrito que, alrededor del 30\% de aquellos que se ven envueltos en un accidente de tránsito, pueden presentar alguna lesión torácica significativa ${ }^{17}$. Asimismo, se puede notar que en nuestro medio el TTC secundario a accidentes de tránsito es más frecuente que en otras realidades. Importante es también mencionar que, los accidentes domésticos, principalmente las caídas a nivel, corresponden en nuestra serie al $14 \%$ de los TTC. Esta información es acorde a otras reveladas por la literatura, donde las caídas alcanzan un $13,8 \%$ de las hospitalizaciones por traumatismo ${ }^{18}$. Creemos que este subgrupo de pacientes, corresponde principalmente a adultos mayores, que, dada su mayor frecuencia de comorbilidades, polifarmacia e inmunosenescencia, están más expuestos a caídas con resultados desfavorablemente más graves.

Uno de los principales factores de riesgo que afecta la mortalidad en el TT es la asociación con lesiones en otros parénquimas. Algunas publicaciones han descrito que el TT se asocia a lesiones extratorácicas hasta en un $25 \%{ }^{18}$. Por el contrario, en nuestra serie, el TTC con lesiones asociadas llegó hasta casi la mitad de los casos (44\%), siendo politraumatizados el 29,6\%. Destacamos que en el subgrupo de los politraumatizados la mortalidad fue de $11,4 \%$.

Las lesiones torácicas producto de TTC pueden dividirse en óseas y de tejidos blandos ${ }^{19}$. En lo que respecta a las óseas, las más comunes son las fracturas costales con un $30-40 \%$ de los $\operatorname{casos}^{5,18,19}$. En pacientes ancianos, las fracturas costales añaden un 19\% de riesgo de mortalidad y un $27 \%$ de desarrollar neumonía ${ }^{7}$. Está descrito que los arcos más afectados son los que van de la tercera a novena costilla. Factores que se han asociado a gravedad de las fracturas costales, son la asociación con lesiones de otras estructuras óseas, fractura de las 2 primeras costillas, más de 3 fracturas costales y la edad avanzada. Especial atención se debe tener en las fracturas costales bajas $\left(9^{\mathrm{a}}-12^{\mathrm{a}}\right)$ ya que no son infrecuentes las lesiones infradiafragmáticas asociadas ${ }^{3}$. No hay que olvidar la asociación de tórax volante con contusión pulmonar, lesión que puede llegar a ser potencialmente grave y letal ${ }^{20}$. Por otro lado, en nuestro medio, en el grupo de pacientes con TTC pudimos observar que las fracturas costales alcanzaron un $75,3 \%$, bastante más de lo descrito, lo que podría corresponder a factores tales como mayor edad de la población y accidentes con mayor energía cinética asociada.

Otros hallazgos torácicos frecuentes fueron en orden decreciente el neumotórax y hemotórax, correspondiéndose a lo descrito en la literatura. Aunque es infrecuente, cabe destacar la posibilidad de asociación de un TTC con lesiones cardiacas, por ejemplo, con la contusión miocárdica que frecuentemente es sub diagnosticada o con entidades como la rotura cardiaca, que hay que sospechar oportunamente, dado el tratamiento quirúrgico precoz que requiere ${ }^{21,22}$. Además, en cuanto a lesiones extratorácicas en pacientes con $\mathrm{TT}$, existen reportes de que las más frecuentes son las de extremidades ${ }^{23}$. Esto es similar en nuestra serie, con $23,3 \%$ lesiones craneoencefálicas asociadas y $16,5 \%$ de extremidades.

En TTC menos del 10-20\% de los pacientes requerirá tratamiento quirúrgico torácico mayor. Se ha descrito que al menos el $60 \%$ de los TT puede ser tratado en el servicio de urgencias, sin requerir siquiera hospitalización ${ }^{18}$. En relación al tratamiento definitivo, la pleurotomía se describe como el de elección en la mayoría de los casos cuando se asocia a hemoneumotórax ${ }^{3}$. La necesidad de cirugía torácica en TT tiene bastante variabilidad en la literatura ${ }^{1,3,5}$, pudiendo deberse al polimorfismo de presentaciones de este grupo 
de pacientes. En nuestra serie, el 8,2\% de los pacientes requirieron cirugía torácica, predominando levemente las cirugías diferidas (después de las primeras $24 \mathrm{~h}$ ) con un $53,2 \%$ del total de las cirugías, seguidas por las urgentes (dentro de las primeras $4 \mathrm{~h}$ ) con un 45,4\%. Las urgentes corresponden principalmente a los sangrados masivos intratorácicos (a causa de hemotórax masivos, lesión de grandes vasos torácicos), mientras que las diferidas corresponden principalmente a complicaciones tardías de los hemotórax (coagulados, retenidos o infectados).

Sobre la morbilidad, hay poca información que se tiene en el grupo de pacientes con TTC en específico. Manay y cols., ${ }^{24}$ en una revisión de 139 TTC, reportaron una morbilidad global de un $30,2 \%$. Importantes son las complicaciones respiratorias que pueden desarrollarse a partir de estos traumatismos. Especialmente en pacientes con fracturas costales en quienes se produce disminución de la expansibilidad del tórax e inestabilidad mecánica-estructural que resulta en una ventilación inadecuada. Esto causa disminución de los volúmenes pulmonares y atelectasias, pudiendo progresar a neumonía o insuficiencia respiratoria. Además, la poca movilización post traumatismo torácico cerrado aumenta la probabilidad de trombosis venosa profunda ${ }^{7}$. Es ahí donde juegan un rol importantísimo la movilización precoz, la kinesioterapia y un adecuado control del dolor para una rehabilitación libre de complicaciones. Por nuestra parte, en esta serie hubo un $17,3 \%$ de casos de morbilidad global.

La mortalidad en pacientes con TT, oscila entre un 4 y $20 \%{ }^{15,23,24}$, sin embargo, en los casos que estos correspondan a pacientes con TTC grave, la mortalidad puede ascender hasta un $40 \%{ }^{24}$. En nuestra serie la mortalidad global alcanzó un 4\%, correspondiendo principalmente a Síndromes de Respuesta Inflamatoria Sistémicos, derivados de TTC asociados a otras lesiones, en particular, en el subgrupo de los politraumatismos.

Los IGT son diferentes puntajes de traumatismo validados y ampliamente utilizados en los principales centros de atención de emergencias en el mundo, que nos indican la gravedad y riesgo de mortalidad, evaluando principalmente parámetros fisiológicos y anatómicos ${ }^{9-11}$. En nuestro medio, observamos que la mortalidad observada es menor a la esperada según IGT.

El TTC tiene un amplio espectro de presentación clínica, abarcando desde los contextos más favorables como, por ejemplo, jóvenes con caídas de nivel con algunas fracturas costales hasta situaciones de extrema gravedad como son los ancianos politraumatizados que derivan de acci- dentes vehiculares de alta energía ${ }^{25}$. Es por esta razón que resulta imperioso dividir y comparar ciertas características fundamentales del traumatismo según si fueron con daño aislado del tórax, si se asociaron a otras lesiones extratorácicas o si se consideraron politraumatismos. Encontramos diferencias estadísticamente significativas entre estos grupos de pacientes.

En conclusión, en nuestro medio, el TTC se presenta más frecuentemente en hombres adultos, a causa de accidentes de tránsito y caídas. La lesión torácica más frecuentemente encontrada son las fracturas costales, predominantemente complicadas con neumotórax y/o hemotórax. La mayoría de los casos se pueden controlar y tratar con medidas conservadoras, ya sea tratamiento médico o pleurotomía. No obstante, no hay que olvidar la importante asociación de los TTC con politraumatismos, lo que lleva a necesitar más tratamientos invasivos y eleva considerablemente la mortalidad.

\section{Bibliografía}

1.- KULSHRESTHA P, MUNSHI I, WAIT R. Profile of Chest Trauma in a Level I Trauma Center. MDJ Trauma 2004; 57: 576-81.

2.- BERNARDIN B, TROQUET JM. Initial management and resuscitation of severe chest trauma. Emerg Med Clin North Am 2012; 30: 377-400.

3.- FREIXINET J, RAMÍREZ M, GALLARDO G, MORENO P. Traumatismos torácicos. Arch Bronconeumol 2011; 47: 9-14.

4.- SIMON LV, BHIMJI SS. Trauma, Blunt Force [Internet]. State pearls. 2018 [citado 25 de abril de 2018]. Recuperado a partir de: https://www.ncbi.nlm.nih.gov/ books/NBK470338/.

5.- GONZÁLEZ R, RIQUELME A, FUENTES A, SALDÍAS R, REYES R, SEGUEL E, et al. Traumatismo torácico: caracterización de hospitalizaciones durante tres décadas. Rev Med Chile 2018; 146: 196-205.

6.- BATtle CE, HUtChings E, EVANS PA. Risk factors that predict mortality in patients with blunt chest wall trauma: a systematic review and meta-analyisis. Injury 2012; 43: 8-17.

7.- UNSWORTH A, CURTIS K, ASHA SE. Treatments for blunt chest trauma and their impact on patient outcomes and health service delivery. Scand J Trauma Resusc Emerg Med 2015; 23: 17.

8.- BAKER SP, O'NEILL B, HADDON W, LONG W. The Injury Severity Score: a method for describing patients with multiple injuries and evaluating emergency care. J Trauma 1974; 14: 187-96.

9.- CHAMPION H, SACCO W, COPES W, GANN D, 
GENNARELLI T, FLANAGAN M. A revision of the Trauma Score. J Trauma 1989; 29: 623-9.

10.- BOYD C, TOLSON M, COPES W. Evaluating trauma care: the TRISS method. Trauma Score and the Injury Severity Score. J Trauma 1987; 27: 370-8.

11.- MICA L, RUFIBACH K, KEEL M, TRENTZ O. The risk of early mortality of polytrauma patients associated to ISS, NISS, APACHE II values and prothrombin time. J Trauma Manag Outcomes 2013; 7: 6.

12.- KEEL M, TRENZ O. Pathophysiology of polytrauma. Injury 2005; 36: 691-709.

13.- ROIS O. Cinemática del trauma. Atención Prehospitalaria Fundamentos; 2004.Disponible en: https:/www. google.com/search?q=Rois+O.+Cinemática + del + trau ma.+Atención+Prehospitalaria+Fundamentos $\% 3 \mathrm{~B}+20$ 04.\&oq=Rois + O. + Cinemática + del + trauma. +

14.- AVARO JP, BONNET PM. The management of blunt chest trauma. Rev Mal Resp 2011; 28: 152-63.

15.- LUDWIG C, KORYLLOS A. Management of chest trauma. J Thorac Dis 2017; 9: 172-7.

16.- HILL AB, FLEISZER DM, BROWN RA. Chest trauma in a Canadian urban setting - implications for trauma research in Canada. J Trauma 1991; 31: 971-3.

17.- DE LESQUEN H, AVARO JP, GUST L, FORD RM, BERANGER F, NATAL EC, et al. Surgical management for the first $48 \mathrm{~h}$ following blunt chest trauma: state of the art (excluding vascular injuries). Interact Cardiovasc Thorac Surg 2015; 20: 399-408.

18.- DONGEL I, COSKUN A, OZBAY S, BAYRAM M, ATIL B. Management of thoracic trauma in emergency service: Analysis of 1139 cases. Pak J Med Sci 2013;
29: $58-63$

19.- ZREIK N, FRANCIS I, RAY A, ROGERS B, RICKETTS D. Blunt chest trauma: bony injury in the thorax. Br J Hosp Med (Lond) 2016; 77: 72-7.

20.- GHYRA A, SANTANDER C, ENRÍQUEZ O, ALARCÓN E, VARGAS A, DOMÍNGUEZ A. Asociación de tórax volante y contusión pulmonar. Análisis de 40 casos. Rev Cir 1993; 45: 609-13.

21.- GONZÁLEZ R, ZALAQUETT R, CHAMORRO G, LEMA G. Rotura de válvula triscúspide con insuficiencia masiva secundaria a traumatismo torácico cerrado. Rev Med Chile 2008; 136: 1034-8.

22.- GONZÁlEZ R, RIQUELME A, FUENTES A, CANALES J, SEGUEL E, STOCKINS A, et al. Traumatismo penetrante cardiaco: caracterización, resultados inmediatos y variables asociadas a morbilidad y mortalidad en pacientes operados. Rev Cir 2019; 71 : 245-52.

23.- AL-EASSA E, AL-MARASHDA M, ELSHERIF A, EID H, ABU-ZIDAN F. Factors affecting mortality of hospitalized chest trauma patients in United Arab Emirates. J Cardiothorac Surg 2013; 8: 57-63.

24.- MANAY P, SATOSKAR R, KARTHIK V, PRAJAPATI R. Studying Morbidity and Predicting Mortality in Patients with Blunt Chest Trauma using a Novel Clinical Score. J Emerg Trauma Shock 2017; 10: 128-33.

25.- FREIXINET J, BELTRÁN J, RODRÍGUEZ P, JULIÁ G, HUSSEIN M, GIL R, et al. Indicadores de gravedad en los traumatismos torácicos. Arch Bronconeumol 2008; 44: 257-62.
Correspondencia a:

Dr. Roberto González Lagos

Departamento de Cirugía, Facultad de Medicina, Universidad de Concepción, Janequeo esquina Chacabuco $\mathrm{S} / \mathrm{N}$, Concepción, Chile.

Email: rgonzalezlagos@udec.cl 